\title{
Effect of Transport Current on the Pinning Induced Magnetostriction of Type-II Superconductors
}

\author{
F. $\operatorname{INANIR}^{a, *}$ AND M. ERDOGAN ${ }^{b}$ \\ ${ }^{a}$ Department of Physics, Rize University, 53100, Rize, Turkey \\ ${ }^{b}$ Department of Physics, Istanbul Technical University \\ 34469, Maslak, Istanbul, Turkey \\ (Received November 2, 2007; revised version November 29, 2007; \\ in final form December 19, 2007)
}

We investigated theoretically the influence of the transport current and applied magnetic field on the magnetostriction of type-II superconductors. In order to study the magnetostrictive response of a superconducting slab specimen, three cases of magnetic history of the sample are considered in the present work: (1) the transport current applied after zero-field-cooling, (2) the current and field are applied simultaneously, (3) the current is applied at the remenent critical state. In the proposed model, the exponential critical state model and the Bean model is employed to compute the flux profile within the slab subjected to sufficiently small transport current and applied magnetic field. Simple expressions for the internal stress and the magnetostriction in terms of this magnetic profile are derived. The magnetostriction curves versus the transport current and applied field are presented.

PACS numbers: 74.25.Ha, 74.25.Ld

\section{Introduction}

In a great deal of technological applications such as ac magnets, power transmission cables, transformers, the superconductors are subjected to the transport current pulses. Dul'kin [1] reported that the value of magnetostriction $\Delta W / W$ of superconducting $\mathrm{YBa}_{2} \mathrm{Cu}_{3} \mathrm{O}_{7-\delta}$ subjected to transport current pulse is of $10^{-4}$, which implies that it is necessary to investigate possible magnetostriction owing to the transport current. The influence of transport current on magnetization of superconductors was studied by LeBlanc [2]. The hysteresis losses due to the transport current flowing through a thin superconducting stripe were first calculated by Norris [3]. The critical-state behavior for an arbitrary sequence of applied

${ }^{*}$ corresponding author; e-mail: inanir@ktu.edu.tr 
transport current was theoretically analyzed by Zeldov et al. [4] for superconducting slab and strip geometries and by Brandt and Indenbom [5] for only strip geometry. The magnetic properties of type II superconductors carrying transport current such as current density, magnetic field, penetrated magnetic flux, magnetic moment, dynamic resistance, AC losses etc. were extensively investigated by others [2-10]. To our knowledge, the pinning induced magnetostriction of a superconductor exposed to transport current has not been studied in the critical-state framework up to date.

When a superconductor is placed into a magnetic field, a noticeable deformation of superconductor occurs in the critical state because of the magnetic force density $\boldsymbol{f}=\boldsymbol{J}_{\mathrm{c}} \times \boldsymbol{B}$, where the current density and $B$ is the magnetic field. As verified by a great number of papers [11-24], this results in an anomalous irreversible magnetostriction and shape distortion in type-II superconductors with strong pinning. The objective of this work is to provide a model to calculate the internal stress and magnetostriction that occurs when a current is transported through a superconductor in absence and presence of the external field in slab geometry within the critical state model. We employ the exponential model which mostly gives a better description of magnetostriction in type II superconductors [11-15].

\section{The magnetostriction of a superconducting slab subjected to a transport current}

In this work, we calculate the magnetostriction hysteresis loops of an infinitely long type-II superconducting slab of thickness $2 W$ as shown in Fig. 1 . We assume the thickness of the slab to be much larger than the London penetration depth $\lambda$ and much smaller than its other two dimensions. The influence of the lower critical field $H_{\mathrm{c} 1}$ and surface effects are neglected in the present treatment. The exponential model for the field dependence of critical current is given by [25, $26]$

$$
J_{\mathrm{c}}(H)=J_{\mathrm{c} 0} \mathrm{e}^{-|H| / H_{0}},
$$

where is the critical current density at zero field and is the field at which $J_{\text {c }}$ reduces to its 1 /e factor. Both are characteristic properties of the material. The Ampère law for the magnetic profile within the slab reads

$$
\frac{\partial H(x)}{\partial x}= \pm J_{\mathrm{c}}(H), \quad 0 \leq x \leq 2 W
$$

where $-(+)$ stands for field increasing (decreasing) process.

We now analyze the response of the specimen to an applied transport current in the positive $y$ direction. To find the magnetic field outside of slab generated by uniform transport current $I_{\mathrm{T}}$, let us consider a rectangular Amperian loop in the $x z$ plane, divided by the sample plane. Applying Ampère's law, we find

$$
\oint \boldsymbol{H} \cdot \mathrm{d} \boldsymbol{l}=2 H l=I_{\mathrm{enc}}=I_{\mathrm{T}} l,
$$

where $l$ is the length of the sides in the rectangle with non-zero contribution to 


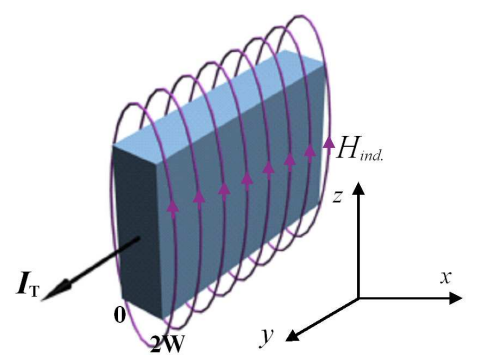

Fig. 1. A superconductor slab with width $2 W$ carrying a transport current $I_{\mathrm{T}}$ in the $y$ direction. $H_{\text {ind }}$ represents the magnetic field induced by the transport current.

the path integral. Therefore $H= \pm I_{\mathrm{T}} / 2$ or more precisely,

$$
\boldsymbol{H}(x)= \begin{cases}-\left(I_{\mathrm{T}} / 2\right) \hat{\boldsymbol{z}} & \text { for } x \leq 0 \\ \left(I_{\mathrm{T}} / 2\right) \hat{\boldsymbol{z}} & \text { for } x \geq 2 W .\end{cases}
$$

Substituting Eq. (1) into Eq. (2) and subjecting to boundary conditions in Eq. (4) $I_{\mathrm{T}} / 2$ at $x=2 W$ and $-I_{\mathrm{T}} / 2$ at $x=$ and also consider the following boundary condition:

$$
\frac{\partial H(x=0)}{\partial x}=\frac{\partial H(x=2 w)}{\partial x}=J_{\mathrm{c}}\left(I_{\mathrm{T}} / 2\right)
$$

we obtain

$$
\int_{I_{\mathrm{T}} / 2}^{-H_{-}(x)} \mathrm{e}^{|H| / H_{0}} \mathrm{~d} H=-\int_{0}^{x} J_{\mathrm{c} 0} \mathrm{~d} x
$$

and

$$
\int_{H_{+}(x)}^{I_{\mathrm{T}} / 2} \mathrm{e}^{|H| / H_{0}} \mathrm{~d} H=\int_{x}^{2 W} J_{\mathrm{c} 0} \mathrm{~d} x
$$

where $H_{-}(x)$ is the field inside the left side of the slab (i.e., $0 \leq x<W_{-}$, see Fig. 2 ) and $H_{+}(x)$, the field inside the right side (i.e., $W_{+}<x \leq 2 W$, see Fig. 2). Such a transport current $I_{\mathrm{T}}$ (per unit length in the $z$-direction) results in a negative flux distribution on the left side of the slab, and a positive flux on the right but in the field free region $J=0$, where the flux does not penetrate, the field is zero. The flux profile in terms of the transport current outside and inside the specimen can be written as

$$
h\left(x^{\prime}\right)= \begin{cases}i, & x^{\prime} \geq 2, \\ \frac{1}{p} \ln \left(\mathrm{e}^{|\mathrm{i}| p}-p\left(2-x^{\prime}\right)\right), & w_{+}<x^{\prime}<2, \\ 0, & w_{-} \leq x^{\prime} \leq w_{+}, \\ -\frac{1}{p} \ln \left(\mathrm{e}^{|\mathrm{i}| p}-p x^{\prime}\right), & 0<x^{\prime}<w_{-}, \\ -i, & x^{\prime} \leq 0,\end{cases}
$$

with $i>0$, where $w_{+}$and $w_{-}$correspond to the flux front positions on the right and on the left of the specimen, respectively, and are found to be 


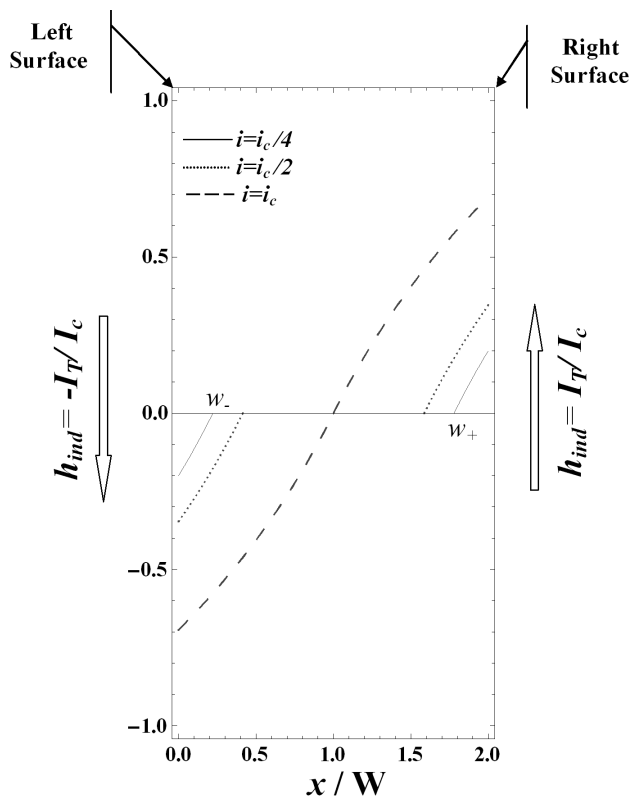

Fig. 2. The calculated magnetic flux density profile inside superconducting slab employing Eq. (8). The transport current $I_{\mathrm{T}}$ is applied after the zero-field cooled process. All values are in the normalized form and $i_{\mathrm{c}}$ is defined by Eq. (14). The profile is $p=1$.

$$
\begin{aligned}
& w_{-}=\frac{1}{p}\left(\mathrm{e}^{|i| p}-1\right), \\
& w_{+}=\frac{1}{p}\left(1+2 p-\mathrm{e}^{|i| p}\right) .
\end{aligned}
$$

Here, the non-dimensional quantities employed are defined by $h\left(x^{\prime}\right) \equiv H(x) / J_{\mathrm{c} 0} W$, $h_{a} \equiv H_{a} / J_{\mathrm{c} 0} W, i \equiv I_{\mathrm{T}} / I_{\mathrm{c}}, I_{\mathrm{c}}=2 J_{\mathrm{c} 0} W, p \equiv J_{\mid m r c 0} W / H_{0}$, and $x^{\prime} \equiv x / W$. where $p$ is also called the pinning parameter determining the field dependence of critical current, defined by Chen et al. [26].

From classical electrodynamics, the force driving the vortices into the material is given by $\mu_{0} \boldsymbol{J} \times \boldsymbol{H}$. In the critical state, equating $J$ to $J_{\mathrm{c}}$ and making use of Eq. (2), the x component of the force exerted on the material per unit volume has been calculated as

$$
F=\frac{\mu_{0}}{2} \frac{\partial H^{2}}{\partial x}
$$

The $x$ component of an internal stress distribution can be obtained by taking the path integral of $F$ along the thickness of the specimen: $\sigma(x)=\int F \mathrm{~d} x$. Finally, we found the stress distribution due to the transport current to be

$$
\sigma_{i}\left(x^{\prime}\right)=\frac{\mu_{0}}{2}\left[H\left(x^{\prime}\right)^{2}-\left(I_{\mathrm{T}} / 2\right)^{2}\right]=\frac{\mu_{0} J_{\mathrm{c} 0}^{2} W^{2}}{2}\left[h^{2}\left(x^{\prime}\right)-i^{2}\right]
$$




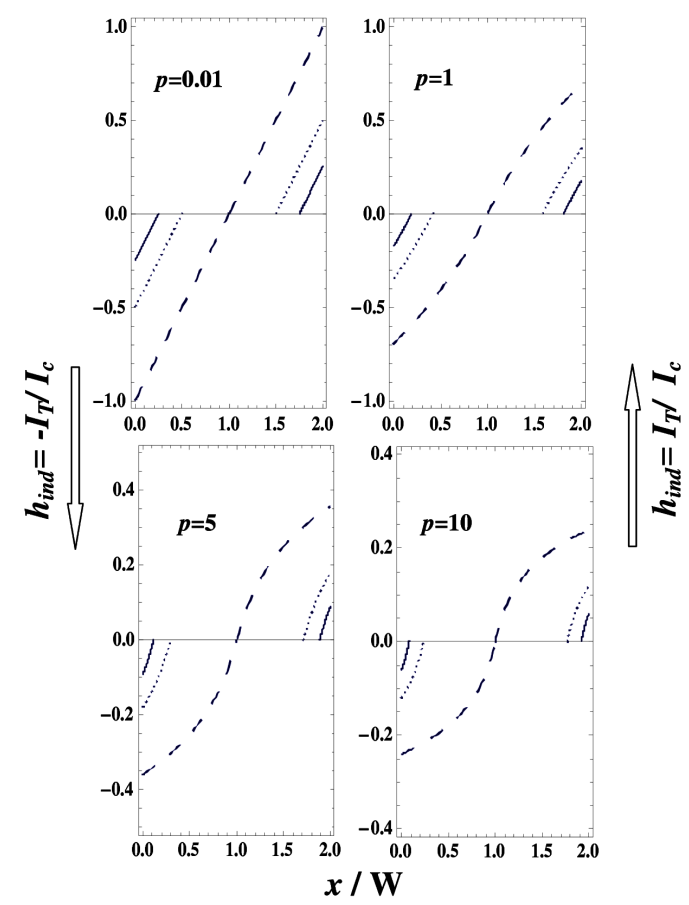

Fig. 3. The simulated magnetic flux distribution inside superconducting slab for various $p$ parameters. The amounts of applied current for each $p$ are $i_{\mathrm{c}} / 4, i_{\mathrm{c}} / 2, i_{\mathrm{c}}$.

$$
=\frac{\mu_{0} J_{\mathrm{c} 0}^{2}}{2} \begin{cases}\frac{1}{p^{2}}\left[\ln \left(\mathrm{e}^{-|i| p}-p\left(2-x^{\prime}\right)\right)\right]^{2}-i^{2}, & w_{+} \leq x^{\prime} \leq 2, \\ -i^{2}, & w_{-} \leq x^{\prime} \leq w_{+}, \\ \frac{1}{p^{2}}\left[\ln \left(\mathrm{e}^{|i| p}-p x^{\prime}\right)\right]^{2}-i^{2}, & 0 \leq x^{\prime} \leq w_{-},\end{cases}
$$

then, magnetostriction reads

$$
\frac{\Delta W}{W}=\frac{1}{2 W} \int_{0}^{2 W} \frac{\sigma(x)}{c_{0}} \mathrm{~d} x=\frac{\mu_{0} J_{\mathrm{c} 0}^{2} W^{2}}{4 c_{0}} \int_{0}^{2}\left[h^{2}(x)-i^{2}\right] \mathrm{d} x,
$$

where $c_{0}$ is the stiffness constant. In the limit of $p \rightarrow 0\left(J_{\mathrm{c} 0} W \ll H_{0}\right)$ the expression yields the Bean result [26-28]:

$$
\lim _{p \rightarrow 0} \frac{\Delta W}{W}=-\frac{\mu_{0} J_{\mathrm{c} 0}^{2} W^{2}}{2 c_{0}} i^{2}==\frac{\mu_{0} I_{\mathrm{T}}^{2}}{8 c_{0}} .
$$

Figure 2 shows the flux-density profiles for the current increasing from zero to $i_{\mathrm{c}}$.

As the current is ascended, the flux front position at left side defined by $W_{-}$slides toward the center of the slab and also, that of the right defined by $W_{+}$ towards center of the slab. The maximum value of $i c$ is determined by equalizing these flux front positions $w_{-}$and $w_{+}$. Thus,

$$
i_{\mathrm{c}}=(1 / p) \ln (1+p) \text {. }
$$

Let us note that $i_{\mathrm{c}}$ is the value at which transport current penetrates the whole 


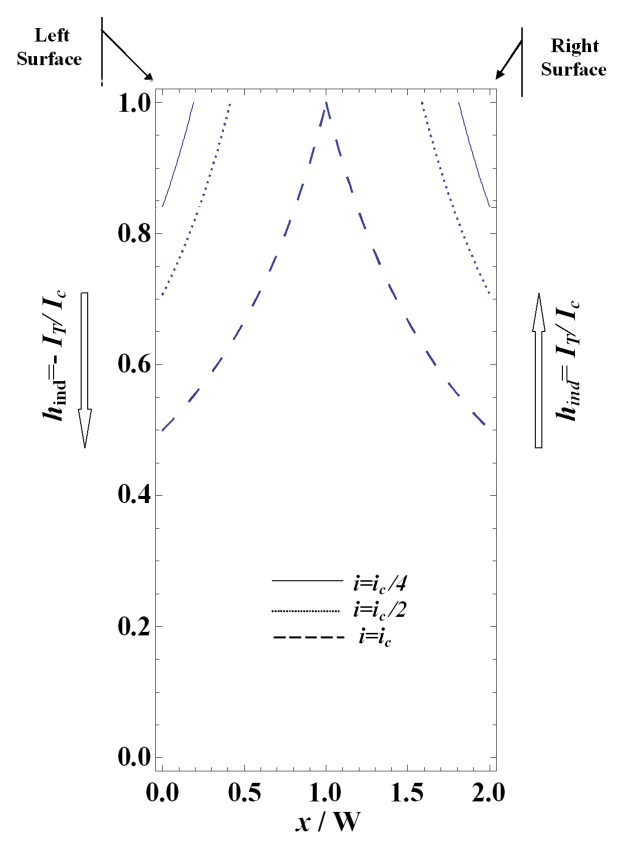

Fig. 4. The calculated current distribution inside slab as the transport current $i=$ $I_{\mathrm{T}} / I_{\mathrm{c}}$ is ascended for the specimen initially in the virgin state. The critical current $I_{\mathrm{c}}$ and total transported current $I$ can be calculated by Eq. (14) and $I=\int_{0}^{2 D} J(x) \mathrm{d} x$, respectively, $p=1$.

slab volume. $I_{\mathrm{c}}$ goes to unity in Bean's limit $p \rightarrow 0$. As long as the applied current flowing within about $\lambda$ of each surface is sufficiently small $(\lambda \ll 2 W)$, so as the slab is in the Meissner state, the magnetic self-field does not penetrate the slab volume, $h(x)=0$ except for $\lambda<x<2 W-\lambda$. When the self-field at the surfaces exceeds the lower critical field $H_{\mathrm{cl}}$, positive flux penetrates the specimen from the right and negative flux infiltrates the left side. It can be deduced from Fig. 3 that the flux distribution inside specimen is strongly dependent on the strength of the field dependence of the critical current. Comparing the profiles of the magnetic field with respect to pinning strength $p$, we see that the field profile is linear for the weak pinning $(p=0.01)$, whereas for the strong pinning the field profile becomes more round. The larger $p$, the more quickly magnetic flux penetrates into the specimen. As a result one observes, with increasing $p$, a decrease in the critical current $i_{\mathrm{c}}$ (given by Eq. (14)). Figure 4 shows the current distribution corresponding to the flux distribution in Fig. 2. The amount of the current transported in the left side and right side of the superconducting slab is equal and at the same direction. The width of the flux-free domain in the slab $w_{+}-w_{-}$depends on both the pinning parameter $p$ and transport current $i$. The width drops exponentially with the applied current and therefore displays nonlinear behavior. 


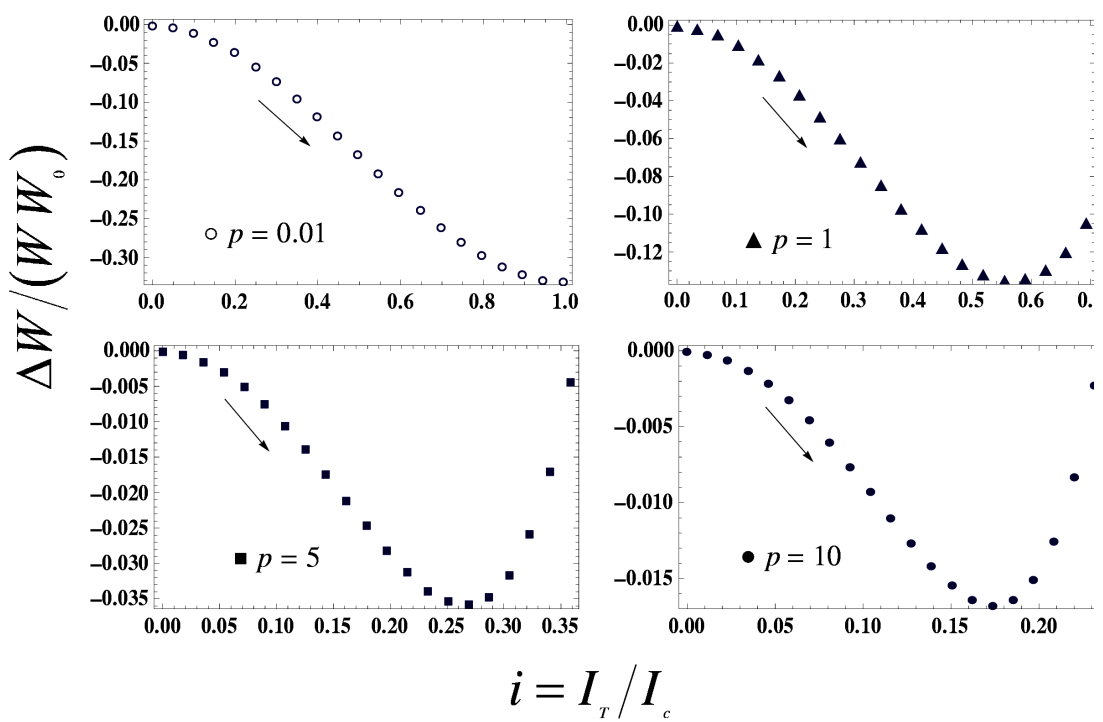

Fig. 5. Calculated magnetostriction curves as a function of the increasing transport current for various pinning parameters $p$. The dilation $\Delta W / W$ and the transport current $I_{\mathrm{T}}$ are normalized by $W_{0}=\mu_{0} J_{\mathrm{c} 0}^{2} W^{2} /\left(4 c_{0}\right)$ and $I_{\mathrm{c}}=2 J_{\mathrm{c} 0} W$, respectively. Maximum transport current value for each $p$ is determined by Eq. (14).

As explained in Ref. [12], according to the exponential model, the parameters that suffice to properly describe the magnetostrictive response of the superconductors in the critical state are $H_{\max }$ and $p$. Here, $H_{\max }$ corresponds to the maximum self-field created by the critical current given by Eq. (14). Figure 5 depicts the magnetostriction curves for various pinning parameters $p$ calculated employing Eq. (12). $\Delta W / W$ is normalized by $W_{0}=\mu_{0} J_{\mathrm{c} 0}^{2} W^{2} /\left(4 c_{0}\right)$. The qualitative features of the curves are not chanced by the normalization since the normalization coefficients affect only the pre-factor in Eq. (12) and thus, it only alters the scale of $\Delta W / W$. As seen in Fig. 5, magnetostriction $\Delta W /\left(W W_{0}\right)$ decreases as $I_{\mathrm{T}}$ increases. This negative magnetostriction of the specimen is owing to the forces exerted by both the positive and negative vortices penetrating the sample. Both shape and scale of the curves strictly depend on the parameter $p$. The behavior of $\Delta W /\left(W W_{0}\right)$ curves does not depend on $p$ at lower current values but starts to vary with $p$ high currents. The local minimum appearing for large $p$ is the characteristic of the exponential model (see Refs. $[12,14]$ ), in which the pinning force $F_{\mathrm{p}}=\mu_{0} \boldsymbol{J} \times \boldsymbol{H}$ versus the self-field has a maximum as parameter $p$ is increased.

3. The magnetostriction calculation of the superconducting slab, subjected to a transport current and a magnetic field

Let us consider the magnetic field $\boldsymbol{H}_{a}=H_{a} \hat{\boldsymbol{z}}$ and the current $\boldsymbol{I}_{\mathrm{T}}=I_{\mathrm{T}} \hat{\boldsymbol{y}}$ applied simultaneously. Using the results in the previous section, a magnetic 


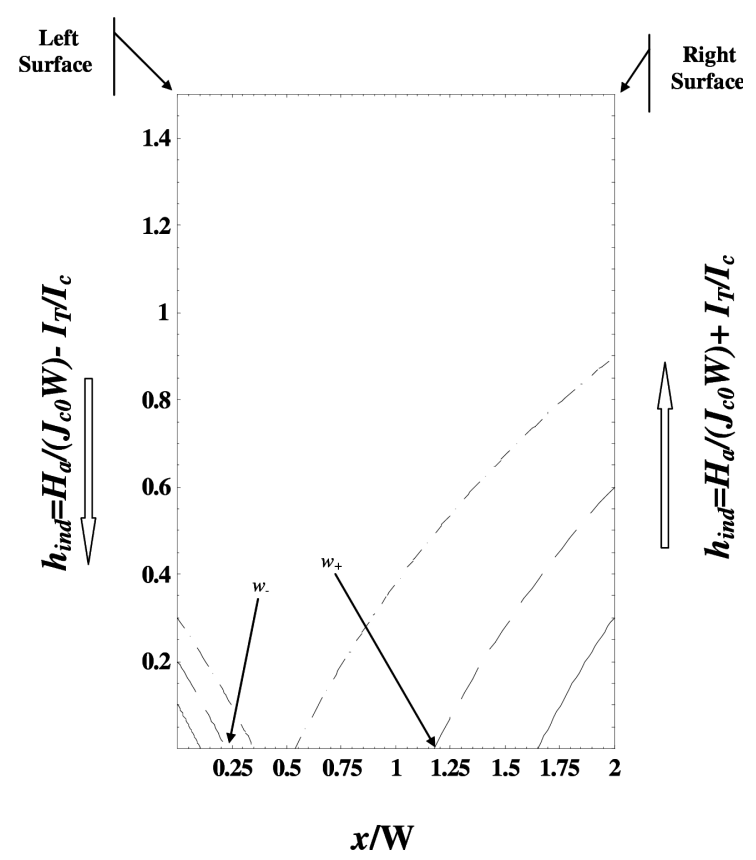

Fig. 6. Simulated flux density profile inside the superconducting slab according to the exponential model $(p=1)$. The field and current are applied simultaneously but the magnitude of applied field outside slab is larger than the magnitude of the self-field induced by the transport current. The arrows indicate the progression of profiles.

field of $\pm I_{\mathrm{T}} / 2$ outside the slab in addition to $H_{a}$ is induced due to the transport current $I_{\mathrm{T}}$.

The resultant field outside of specimen is given by

$$
\boldsymbol{H}(x)= \begin{cases}\left(H_{a}-I_{\mathrm{T}} / 2\right) \hat{\boldsymbol{z}}, & x \leq 0, \\ \left(H_{a}+I_{\mathrm{T}} / 2\right) \hat{\boldsymbol{z}}, & x \geq 2 W .\end{cases}
$$

Inserting Eq. (1) into Eq. (2) and exploiting field values at the specimen edges $H_{a}+I_{\mathrm{T}} / 2$ at $x=0$ and $H_{a}-I_{\mathrm{T}} / 2$ at $x=2 W$ given by Eq. (15) (Fig. 6), the field inside the left side of slab $H(x)$ is calculated using the following integrals:

$$
\int_{H_{a}-\frac{l_{\mathrm{T}}}{2}}^{H_{-}(x)} \mathrm{e}^{|H| / H_{0}} \mathrm{~d} H=-\int_{0}^{x} J_{\mathrm{c} 0} \mathrm{~d} x
$$

and the field inside the right side of slab $H(x)$.

$$
\int_{H_{+}(x)}^{H_{a}+\frac{l_{\mathrm{T}}}{2}} \mathrm{e}^{|H| / H_{0}} \mathrm{~d} H=\int_{x}^{2 W} J_{\mathrm{c} 0} \mathrm{~d} x
$$

The magnetic profile expression in the reduced form outside and inside the slab due to the transport current and the applied magnetic field is given more compactly, 


$$
h\left(x^{\prime}\right)= \begin{cases}h_{a}+i & x^{\prime} \geq 2, \\ \frac{1}{p} \ln \left(\mathrm{e}^{\left|h_{a}+\mathrm{i}\right| p}-p\left(2-x^{\prime}\right)\right), & w_{+}<x^{\prime}<2, \\ 0, & w_{-} \leq x^{\prime} \leq w_{+}, \\ \frac{1}{p} \ln \left(\mathrm{e}^{\left|h_{a}-\mathrm{i}\right| p}-p x^{\prime}\right), & 0<x^{\prime}<w_{-}, \\ h_{a}-i, & x^{\prime} \leq 0,\end{cases}
$$

with the flux fronts

$$
w_{-}^{\prime}=\frac{1}{p}\left(1-\mathrm{e}^{\left|h_{a}-i\right| p}\right), \quad w_{+}^{\prime}=\frac{1}{p}\left(1+2 p-\mathrm{e}^{\left|h_{a}+i\right| p}\right)
$$

on the left and on the right, respectively. The field distribution obtained in Eq. (18) is shown in Fig. 6. Such field profile can be carried out assuming that the magnitude of the applied field outside of specimen must be larger than the magnitude of the self-field induced by the transport current, $h_{a} \geq i$. However, the ascending rate of the applied field must be greater than that of the current, $\mathrm{d} h_{a} / \mathrm{d} t \geq \mathrm{d} i / \mathrm{d} t$.

We calculated the stress distribution created by the magnetic profile given in Eq. (18) as

$$
\begin{aligned}
& \sigma_{i, h}\left(x^{\prime}\right)= \\
& =\frac{\mu_{0} J_{\mathrm{c} 0}^{2}}{2} \begin{cases}\frac{1}{p^{2}}\left[\ln \left(\mathrm{e}^{\left|h_{a}+i\right| p}-p\left(2-x^{\prime}\right)\right)\right]^{2}-\left(h_{a}+i\right)^{2}, & w_{+}^{\prime} \leq x^{\prime} \leq 2, \\
-\left(h_{a}+i\right)^{2}, & w_{-}^{\prime} \leq x^{\prime} \leq w_{+}^{\prime}, \\
\frac{1}{p^{2}}\left[\ln \left(\mathrm{e}^{\left|h_{a}-i\right| p}-p x^{\prime}\right)\right]^{2}-\left(h_{a}+i\right)^{2}, & 0 \leq x^{\prime} \leq w_{-}^{\prime} .\end{cases}
\end{aligned}
$$

The boundary values of the stress function $\sigma_{i, h}(x=2 W)=-\mu_{0} H_{a} I_{\mathrm{T}}$ and $\sigma_{i, h}(x=$ $0)=0$ imply an unbalanced force acting on the slab. The stress value at $x=2 W$ is nothing else but the path integral of the Lorentz force along the sample thickness. This force makes the slap to move in the positive $x$ direction and makes it suffer from an inertial force in non-inertial frames of reference leaving the boundary free of stress. The magnetostriction can be calculated inserting the stress expression Eq. (20) into Eq. (12) except the additional term coming from the inertial force. Taking the Bean limit $p \rightarrow 0$, we have

$$
\frac{\Delta W}{W}=\frac{\mu_{0} J_{\mathrm{c} 0}^{2} W^{2}}{2 c_{0}}\left[\frac{1}{3}\left(h_{a}-3\right) h_{a}^{2}-6 h_{a} i+\left(h_{a}-1\right) i^{2}\right] .
$$

In absence of applied magnetic field, i.e. $h_{a}=0$ this expression transforms back into Eq. (13).

\section{The magnetostriction analysis of a superconductor carrying transport current applied at remenent state}

We have analyzed the field distribution produced when a transport current is applied to a specimen in the remanent state. The remanent state is described as the critical state that is created by initially subjecting the specimen to a very high magnetic field, at least larger than twice the full penetration, and then decreasing 


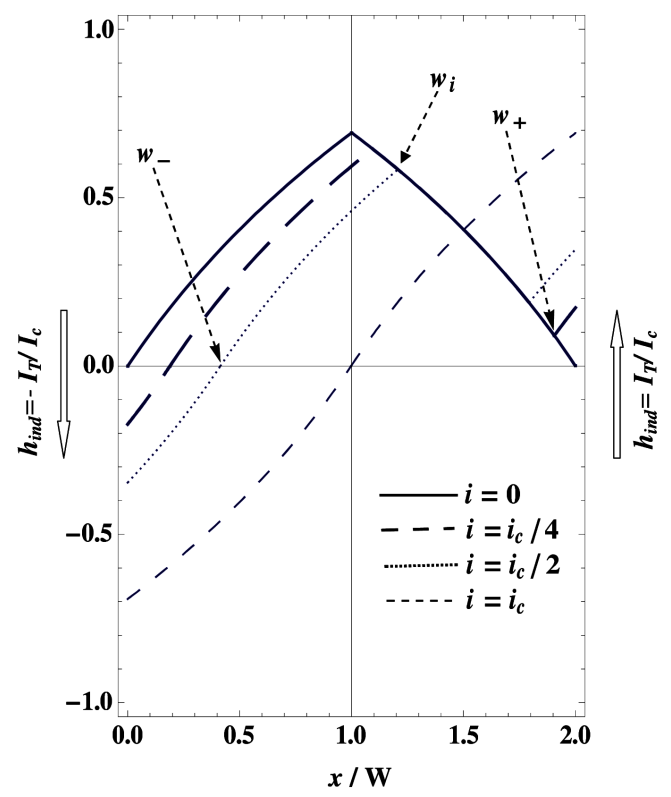

Fig. 7. Evolution of magnetic flux profile calculated employing exponential model as the transport current $I_{\mathrm{T}}$ is ascended for a slab sample initially in the fully penetrated remenent state. The solid curve shows the initial $I_{\mathrm{T}}=0$ profile, and arrows imply the progression of the subsequent profile as $I_{\mathrm{T}}$ increased, $p=1$.

the applied field to zero. A transport current is gradually applied in the $y$ direction after the remenent state. As seen in Fig. 7, this additional current alters the magnetic field profile and hence the vortex distribution. The current causes a flux penetration from the right side and a flux escapes from the left side of the specimen. Flux profile equations described in this state are given by

$$
h\left(x^{\prime}\right)= \begin{cases}-\frac{1}{p} \ln \left(\mathrm{e}^{|i| p}+p x^{\prime}\right), & 0 \leq x^{\prime} \leq w_{-}, \\ \frac{1}{p} \ln \left(1+p\left(x^{\prime}-w_{-}\right)\right), & w_{-} \leq x^{\prime} \leq w_{i}, \\ \frac{1}{p} \ln \left(1+p\left(2-x^{\prime}\right)\right), & w_{i} \leq x^{\prime} \leq w_{+}, \\ \frac{1}{p} \ln \left(\mathrm{e}^{|i| p}-p\left(2-x^{\prime}\right)\right), & w_{+} \leq x^{\prime} \leq 2 .\end{cases}
$$

Continuity of $h\left(x^{\prime}\right)$ yields the following relations:

$$
w_{+}=\frac{1}{2 p}\left(1+4 p-\mathrm{e}^{|i| p}\right), \quad w_{-}=\frac{1}{p}\left(1-\mathrm{e}^{|i| p}\right), \quad w_{i}=1+\frac{1}{2} w_{-} .
$$

Figure 8 shows how $\Delta W /\left(W W_{0}\right)$ depends on the transport current for different values of parameter $p$. The figure was obtained by substituting Eq. (22) into Eq. (12). Both $\Delta W / W$ and $I_{\mathrm{T}}$ are again represented in the normalized form, as above. Owing to the trapped flux lines trying to leave the specimen, the superconducting slab in the remenent state is expanded compared to the original, zero-field cooled length. The extension of the sample in this state is given by 


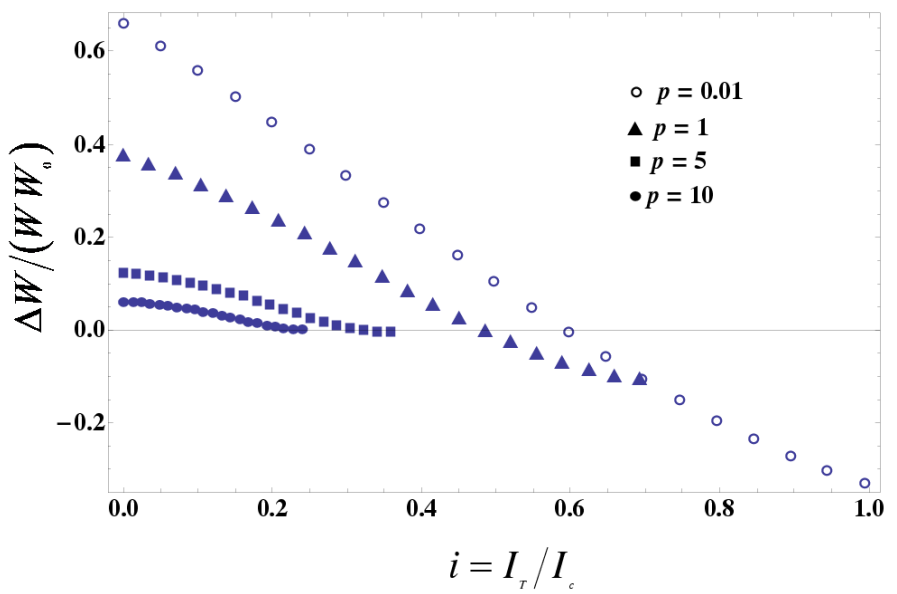

Fig. 8. Family of curves of magnetostriction versus different pinning parameter $p$, as a current is transported after fully remenent state. Here $W_{0}=\mu_{0} J_{\mathrm{c} 0}^{2} W^{2} /\left(4 c_{0}\right)$ and $I_{\mathrm{c}}=2 J_{\mathrm{c} 0} W$

$$
\left(\frac{\Delta W}{W W_{0}}\right)_{\mathrm{REM}}=\frac{2 p+(1+p)(-2+\ln (1+p)) \ln (1+p)}{p^{3}} .
$$

When the transport current is ascended, one sees that the dilation $\Delta W /\left(W W_{0}\right)$ gradually decreases and the sample reaches the original length and then begins to contract. The contraction for $p=10$ is very small in comparison with $p=0.01$ and 1 . This arises from that the amount of the vortices that penetrate and exit through edge of the sample is controlled by the pinning parameter $p$.

\section{Conclusion}

We have presented a general approach in the frame of the critical state model to calculate the complex magnetostriction hysteresis of the superconducting sample subjected to a transport current with and without magnetic field. In this modeling work, we have utilized an exponential type decay $J_{\mathrm{c}}=J_{\mathrm{c} 0} \mathrm{e}^{-|H| / H_{0}}$ of critical current density. The transport current can induce a negative magnetostriction. We note that investigation of transport current effect on type-II superconductors' magnetostriction can offer an alternative tool to study their flux dynamic properties and also becomes important for technological aspect.

\section{References}

[1] E.A. Dul'kin, Tech. Phys. Lett. 26, 565 (2001).

[2] M.A.R. LeBlanc, Phys. Rev. Lett. 11, 149 (1963).

[3] W.T. Norris, J. Phys. D 3, 489 (1970).

[4] E. Zeldov, J.R. Clem, M. McElfresh, M. Darwin, Phys. Rev. B 49, 9802 (1994).

[5] E.H. Brandt, M. Indenbom, Phys. Rev. B 48, 12893 (1993). 
[6] A.V. Bobyl, D.V. Stantsev, Y.M. Galperin, T.H. Johansen, M. Baziljevich, S.F. Karmanenko, Supercond. Sci. Technol. 15, 82 (2002).

[7] M.P. Oomen, J. Rieger, M. Leghissa, B. ten Haken, H.H. J. ten Kate, Supercond. Sci. Technol. 12, 382 (1997).

[8] J.L. Giordino, L.A. Angural, Supercond. Sci. Technol. 14, 655 (2001).

[9] A. M. Campbell, J.E. Evetts, Adv. Phys. 50, 1249 (2001) and some references therein.

[10] J.C. van der Beek, G.J. Nieuwenhuys, P. Kes, H.G. Schnack, R.P. Griessen, Physica C 192, 403 (1992).

[11] H. Ikuta, N. Hirota, Y. Nakayama, K. Kishio, K. Kitazawa, Phys. Rev. Lett. 70, 2166 (1993).

[12] H. Ikuta, K. Kishio, K. Kitzawa, J. Appl. Phys. 76, 4776 (1994).

[13] S. Çelebi, F. Inanir, M.A.R. LeBlanc, Supercond. Sci. Technol. 18, 14 (2005).

[14] F. Inanir, S. Çelebi, J. Alloys Comp. 427, 1 (2007).

[15] F. Inanir, S. Çelebi, M. Atunbas, M. Okutan, M. Erdogan, Physica C 459, 17 (2007).

[16] K. Flber, A. Geerkens, S. Ewert, K. Winzer, Physica C 299, 1 (1998).

[17] A. Nabiałek, H. Szymczak, K. Poitrowski, V. Chabanenko, Z. Pakieła, Physica C 321, 49 (1999).

[18] V.V. Eremenko, V.A. Sirenko, R. Schleser, P.L. Gammel, Low Temp. Phys. 27, 305 (2001).

[19] A. Gerber, R. Schleser, J.E.M. van der Linden, P. Wyder, Physica B 319, 293 (2002).

[20] A. Nabiałek, B. Kundys, Yu Bukhantsev, S. Vasiliev, A. Wiśniewski, J. Jun, S.M. Kazakov, J. Karpiński, H. Szymczak, Physica B 319, 286 (2002).

[21] A. Nabiałek, B. Kundys, Yu Bukhantsev, A. Wiśniewski, S.M. Kazakov, J. Karpinski, H. Szymczak, Supercond. Sci. Technol. 16, 707 (2003).

[22] A. Nabiałek, H. Szymczak, V.V. Chabanenko, J. Low Temp. Phys. 139, 309 (2005).

[23] T.H. Johansen, Supercond. Sci. Technol. 13, R121 (2000).

[24] T.H. Johansen, Phys. Rev. B. 59, 11187 (1999).

[25] W.A. Fietz, M.R. Beasley, J. Silcox, W.W. Webb, Phys. Rev. 136, A335 (1964).

[26] D.-X. Chen, A. Sanchez, J.S. Munoz, J. Appl. Phys. 67, 3430 (1989).

[27] C.P. Bean, Phys. Rev. Lett. 8, 250 (1962).

[28] C.P. Bean, Rev. Mod. Phys. 36, 31 (1964). 